\title{
Wind Power and Electricity Consumption Forecasting on a Smart House Location
}

\author{
H. Eliasstam ${ }^{1}$, K. N. Genikomsakis ${ }^{2}$ and C. S. Ioakimidis ${ }^{2 *}$ \\ ${ }^{1}$ Department of Applied Physics and Electrical Engineering \\ Linköping University \\ SE-581 83, Linköping (Sweden) \\ e-mail: hannes.eliasstam@gmail.com \\ ${ }^{2}$ Deusto Institute of Technology, DeustoTech Energy \\ University of Deusto \\ Avenida de las Universidades 24, 48007 Bilbao (Spain) \\ *Phone/Fax number: +34 944139000 (x 2704) / +34 944139101, e-mail: kostas.genikomsakis@deusto.es, \\ christos.ioakimidis@deusto.es
}

\begin{abstract}
This paper presents the use of an artificial neural network for classification on a residence house that uses wind and electricity consumption predictions to identify patterns at the desired location, in order to obtain a stochastic distribution of the daily wind and electricity profile. This is a step on the further creation of a short-term operation model that allows determining the technical and economic impact of stationary/mobile batteries of electric vehicles in presence of microrenewables along with the electricity consumption. This short-term operation model will be in the day-ahead perfect market operation (unit commitment) where specific changes are made to consider stationary and mobile operation.
\end{abstract}

\section{Key words}

Artificial neural networks, Smart homes, Forecasting, Wind energy, Electricity

\section{Introduction}

The owners of a household in today society do not know in a real-time how much electricity they use. It usually takes about three months before the owners receive the bills and realize that they used too much electricity. It would be comfortable to have more control and overview in realtime over the electricity consumptions and the cost of a house. This could be possible with a system that monitors the consumptions, micro generations (i.e., wind and solar) and the electricity prices from the grid and then make decisions every few minutes (i.e., 10 minutes) to make a profit for the household.

This kind of system manages different storage devices i.e. a fix battery in the basement and an electric vehicle (EV) when it is plugged in for charging. The EV battery should be enough charged before the owner head to go to work. The system manages and optimizes the energy to make a profit for the owner, i.e. minimize the costs or maximize the income.

The idea is to apply this kind of system to multiple houses in a certain area. This group of houses can work as a mini supplier of electricity to the grid through an aggregator (i.e., Energy Box) placed in the area by a contractor. The aggregator controls the amount and the frequency of energy that enter the grid to avoid overflow and blackouts. This kind of mini supplier can enter the grid through an aggregator to sell energy during peak hours. It may be a problem during the peak hours that there is a higher demand than the demand calculated by the electricity suppliers. To meet these demands the electricity companies need to start new generators which could be expensive and takes time. Instead the mini suppliers can meet these extra demands on a very short notice from their storage devices. This results in less cost to the electricity company and a profit for the individual household, thus a win-win situation.

Considering the possibility of having energy produced by local microrenewables at the residence, and storage capacity provided from electric vehicle's battery, the energy box needs to have as an input the forecasted wind and solar predictions in order to estimate the electricity production during the day, to then decide to sell or buy, and charge or discharge the EV's battery. For the energy box we consider a horizon of 24 hours in what the future states influence the present decision, so it is necessary to forecast the wind profile in that period. In order to do so, it is used as an input the local meteorological predictions and as output we obtain the discrete probability distribution of the wind profile at the desired location. To obtain this output it is proposed to use an Artificial Neural Network (ANN), a mathematical model that tries to simulate the behaviour of biological neural networks, such as the human brain. 


\section{Smart house and the microgrid architecture}

The microgrid architecture (Fig. 1) of the corresponding Smart House can be multiple microgrids when a number of houses, can be connected to the national main grid (or macrogrid), or operate on a disconnected (islanded) mode if problems arise from the main grid [1], and reconnected once they are solved. It is possible to obtain several microgrid configurations that may contain renewable and non-renewable energy sources, electrical energy storage capacity and remote controlled loads. Several academics and researchers investigated these configurations for specific locations as in $[2,3]$.

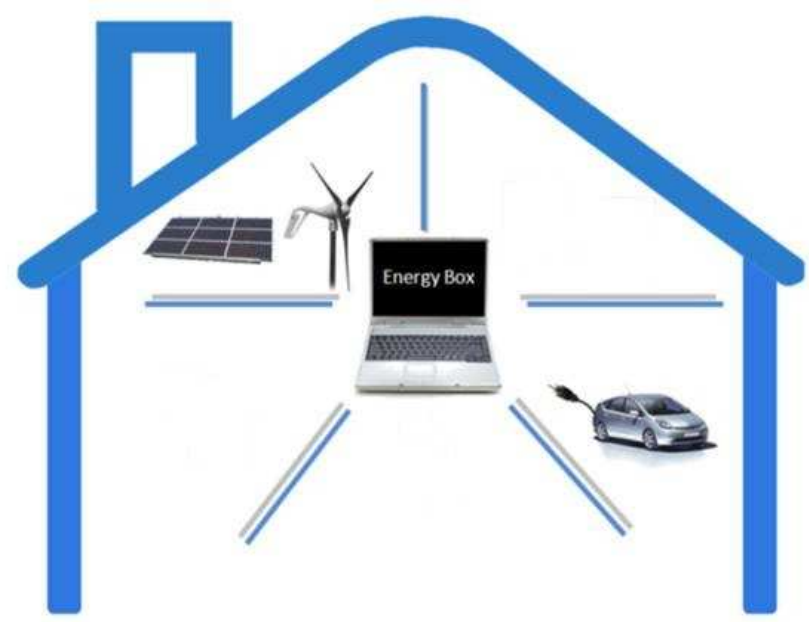

Fig. 1. The Residential Smart House Aggregator (Energy Box) Paradigm.

\section{Subsystem description}

In this section, the mathematical models along with their reference for each subsystem will be presented and wherever possible, a comparison between simulated results and manufacturer's data or experimental results will be given. The simulation and theoretical study was achieved by using Matlab®.

\section{A. Wind system}

The wind turbine converts the kinetic energy of the wind into rotational energy and then into electrical energy. The definition of a kinetic energy of an object with mass $m$ and velocity $\mathrm{v}$ is given by (1)

$\mathbf{E}=\frac{\mathbf{m} * \mathbf{v}^{2}}{2}$

The power produced by the wind is given by the energy flow rate:

$P_{\text {wind }}=\frac{\mathrm{dE}}{\mathrm{dt}}=\frac{\mathrm{v}^{2}}{2} * \frac{\mathrm{dm}}{\mathrm{dt}}$,

as the mass flow rate is given by

$\frac{\mathrm{dm}}{\mathrm{dt}}=\boldsymbol{\rho} * \mathbf{A} * \frac{\mathrm{dx}}{\mathrm{dt}}=\boldsymbol{\rho} * \mathbf{A} * \mathbf{v}$

Inserting equation (2) into equation (3), gives
$\mathbf{P}_{\text {wind }}=\frac{\mathrm{dE}}{\mathrm{dt}}=\frac{\mathbf{v}^{2}}{2} * \boldsymbol{\rho} * \mathbf{A} * \mathbf{v}=\frac{\rho * \mathrm{~A}}{2} * \mathbf{v}^{3}$

Every wind turbine has a specific power curve due to different manufacturing. This indicates that a wind turbine cannot extract all the energy from the wind and a power coefficient has to be included in the above mentioned equation.

$\mathbf{P}_{\text {wind }}=\frac{\rho * \mathbf{A}}{2} * \mathbf{C}_{\mathbf{p}} * \mathbf{v}^{3}$, where

$\rho=1.225\left[\frac{\mathrm{kg}}{\mathrm{m}^{3}}\right]$, the air density

$\boldsymbol{A}=\boldsymbol{\pi} * \boldsymbol{r}^{2}\left[\mathbf{m}^{2}\right]$, the swept area of the turbine, $\boldsymbol{r}$ is the radius.

$v\left[\frac{m}{s}\right]$, the wind speed

$\mathbf{C}_{\mathbf{p}}$, the power coefficient.

However there is a theoretical maximum value of the power coefficient [4] $\boldsymbol{C}_{\boldsymbol{p}}=\frac{\mathbf{1 6}}{\mathbf{2 7}} \approx \mathbf{0 . 5 9}$, which has been calculated by the German physicist Albert Betz in 1919. In reality the wind turbines achieves peak values for $\boldsymbol{C}_{\boldsymbol{p}}$ in the range of 0.40 to 0.50 (about $68 \%$ to $85 \%$ of the theoretically possible maximum) due to profile loss, tip loss and loss due to wake rotation. Also, in high wind speed where the turbine is operating at its rated power the turbine rotates (pitches) it is blades to lower $\boldsymbol{C}_{\boldsymbol{p}}$ to protect itself from damage and in order to determine the mechanical power available for the load machine (electrical generator, pump).

The wind turbine model Anern 1000L [5] is considered in the modeling. This model has a radius of $1.9[\mathrm{~m}]$, cutin speed of $3\left[\frac{m}{s}\right]$ (at this wind speed the turbine start to generate energy) and cut-out speed of $18\left[\frac{m}{s}\right]$ (at this wind speed a braking system is employed on the turbine to not damage the rotor). Figure Fig. below demonstrates the power curve [4].

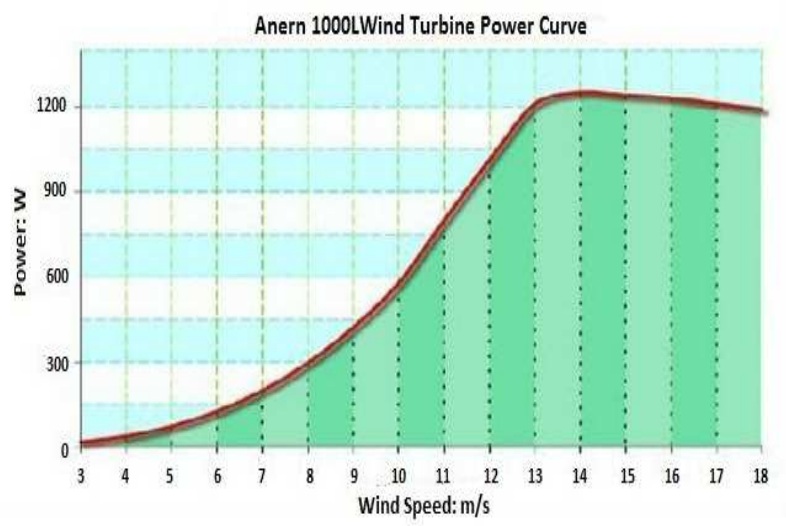

Fig. 2. The power curve of Anern 1000L.

Since there is a limit of mechanical and electric generation the power curve has the shape shown in figure 
2 above. Different $\boldsymbol{C}_{\boldsymbol{p}}$ values calculated for different wind speeds backward according to the formula below:

$C_{p}=\frac{P_{\text {anern }}}{0.5 * \rho * A * v_{\text {anern }}^{3}}$

This is done through a lookup table that has values of the power curve for every $0.5\left[\frac{m}{s}\right]$. The actual wind speed $v$ between 3 and $18\left[\frac{m}{s}\right]$ is rounded to the closest 0.5 value (the rounded speed becomes $\boldsymbol{v}_{\text {Anern }}$ ) and for every 0.5 value there is a corresponding power value $\left(\boldsymbol{P}_{\text {anern }}\right)$. $\boldsymbol{P}_{\text {anern }}$ is set to zero for the wind speed less than 3 and more $18\left[\frac{\mathrm{m}}{\mathrm{s}}\right]$. Now the output power of Anern $1000 \mathrm{~L}$ can be calculated according to equation (5).

$\mathbf{P}_{\text {anern,tot }}=\mathbf{N}_{\text {anern }} * \mathbf{P}_{\text {anern }}$, where

$\mathbf{N}_{\text {anern }}=2$, the amount of turbines used here.

\section{A. Electricity consumption and prices}

Every house has its daily consumption of electricity and it varies from country to country. The house consumption varies as well throughout the day and during different seasons. Since the study is done in Bilbao, Spain, the consumptions are considered for a single household in Spain of four people, a couple with two children. According to the consumption profile for the year 2010, the household consumption is approximately $17.7 \mathrm{KW}$ during a day. For this work, a consumption profile for the year 2011 is used. The consumption profile is based on the electricity consumption for the past few years and calculates a consumption coefficient. This is then multiplied by the average total consumption for the whole year.

It is important for this work to know the electricity prices for buying and selling the energy. The prices are determined through a contract with the supplier and the prices are listed in $€ / \mathrm{kWh}$. According to the consumption profile for the year 2010, the yearly consumption of the household is ca $6470 \mathrm{~kW} / \mathrm{year}$. The consumption in this case is every 10 minutes, the energy would be 6470 $\mathrm{kW} 10$ min/year, given that Energy=power*time. This gives ca $1080 \mathrm{kWh} /$ year, however it is normal that the supplier gives different prices for $\mathrm{kWh}$ depending on how much energy every household use. According to the Europe's Energy Portal [6], the energy cost in Spain is 0.2013 $€ / \mathrm{kWh}$ for households that use up to $3500 \mathrm{kWh} /$ year compared to $0.1839 € / \mathrm{kWh}$ for households that use up to $7500[\mathrm{kWh} /$ year]. The electricity supplier usually offers different contracts that include variable or fix prices, while some suppliers offer a mix of both variable and fix prices.

As mentioned before, the electricity prices in the Spanish market are regulated by RED ELĖCTRICA DE ESPAÑA (REE). REE calculates the sell prices for the 24 hours ahead bidding market, which are set for every hour. In Fig. 3 shows the prices for one week of 24 hours ahead bidding market.

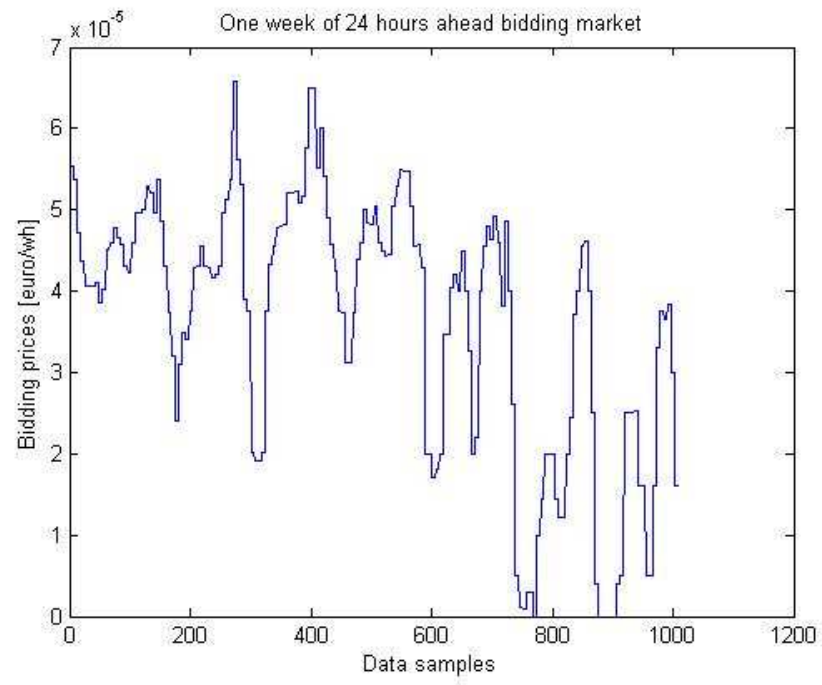

Fig. 3. One week of 24 hours ahead bidding market.

These prices are based on the demand in the market and some of the prices are set to zero. This means that there is no demand at all, i.e. the market is saturated with energy and there is no bidding. Since the prices that REE offers are the prices of the next 24 hours, there is no need to estimate them. The sell prices ( $€ / \mathrm{W} 10 \mathrm{~min})$ for the year 2011 are used in this work. A contracted company and what type of contract should be chosen to get the buy prices. The Iberdrola electric utility company is considered as the electricity supplier. Iberdrola offers peak and valley prices as well winter and summer time table [7]. The peak prices correspond to 10 hours a day, from $12 \mathrm{~h}$ to $22 \mathrm{~h}$ winter time and from $13 \mathrm{~h}$ to $23 \mathrm{~h}$ summer time. The valley prices are 14 hours, from $0 \mathrm{~h}$ to $12 \mathrm{~h}$ also from $22 \mathrm{~h}$ to $24 \mathrm{~h}$ winter time and from $0 \mathrm{~h}$ to $13 \mathrm{~h}$ also from $23 \mathrm{~h}$ to $24 \mathrm{~h}$ summer time. The time table changes with the official clock change, for the year 2011 that is on the 27th of March for summer time and on the 30th of October for winter time. The electricity prices [8] are updated twice a year. For the first 6 months of the year 2011 , the prices are $0.168743 € / \mathrm{kWh}$ for peak and $0.06089 € / \mathrm{kWh}$ for valley. For the last 6 months of the year 2011 , the prices are $0.17282 € / \mathrm{kWh}$ for peak and $0.064047 € / \mathrm{kWh}$ for valley. This data is inserted in MATLAB as $€ / W 10 \mathrm{~min}$.

\section{Artificial Neural Networks (ANN)}

An artificial neural network (ANN) [9] is a mathematical model that is inspired by the structure and functional aspects of biological neural networks, like the human brain. A neural network consists of an interconnected group of artificial neurons. In most cases an ANN is an adaptive system that changes its structure based on external or internal information that flows through the network during the training phase. Advanced neural networks are non-linear statistical data modeling tools. They are usually used to model complex relationships between inputs and outputs or to find patterns in data. Even though artificial neurons are simplified, they can show a variety of input-output relations, depending on the transfer functions they apply. The different choice or 
combination of transfer functions gives different behavior and fits different types of problems.

The ANN consists of layers, the first layer has input neurons, which send data via synapses to the second layer of neurons (one hidden layer is standard in MATLAB, more than one hidden layers can be used) and then via more synapses to the third layer, which includes the output neurons. More complex systems have more hidden layers with increased number of input and output neurons. The synapses store parameters called weights that manipulate the data in the calculations.

An ANN is typically defined by three types of parameters:

1. The interconnection pattern between different layers of neurons.

2. The learning process for updating the weights of the interconnections.

3. The activation function that converts a neuron's weighted input to its output activation.

Figure 4 below illustrates the different layers.

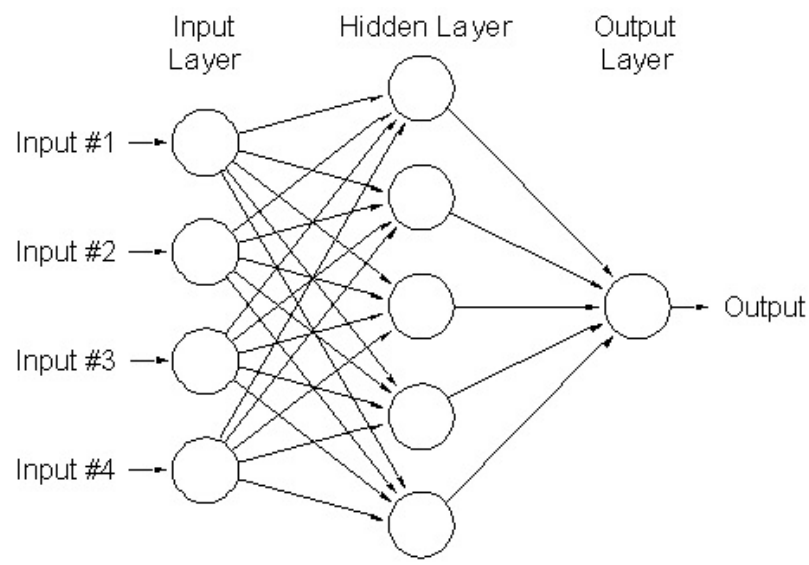

Fig. 4. Artificial Neural Network (ANN).

\section{Data Collection}

\section{A. Test location}

Some data is needed for the project. The wind speed is needed for the wind turbine. The electricity consumptions are needed to know how much electricity consumes the household and the electricity prices are needed to know how much the electricity costs in the grid. In this work the data of wind velocity air temperature, solar insulation, has been collected through a local weather station [10], and is updated every 10 minutes. Therefore it would be natural to construct a system that makes decision every 10 minutes. The data was downloaded as excel files and then fetched to MATLAB. The outliers had to be corrected and an interpolation had to be done since some of the data was missing.

\section{B. Preprocessing data and estimation}

Obtaining and processing data to train the Neural Network is an essential part for the success and quality of the model's predictions. All of the predictions are done with the Artificial Neural Network (ANN) tool in MATLAB which is based on the measured meteorological data from the year 2010 in the city of Bilbao. The Neural Network fitting tool is used and since the prediction is for the next 24 hours, the data is divided (day 1 till day 364) as input and (day 2 till day 365) as target. Then we divide the data as $70 \%$ training data, $15 \%$ validation data and $15 \%$ testing data.

In this case the network will be trained with the function Levenberg-Marquardt backpropagation algorithm (called trainlm), unless there is not enough memory then the scaled conjugate gradient backpropagation algorithm will be used (called trainscg). In this network one hidden layer and one output layer is used, as shown in Fig 5.

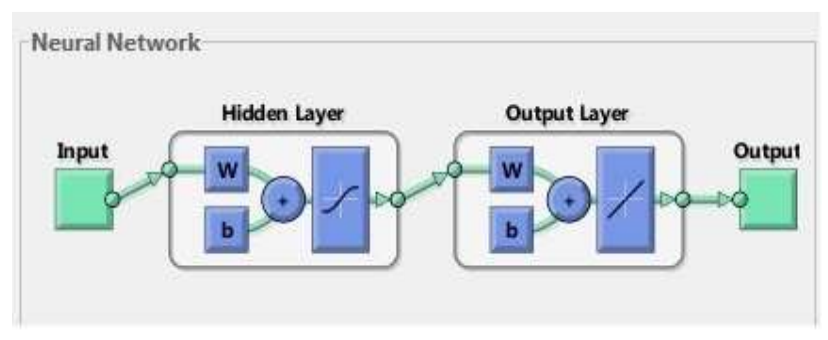

Fig. 5. Neural Network layers fitting tool.

The hidden layer has 10 neurons with Tan-Sigmoid transfer function and the output layer has 1 neuron with Linear transfer function, (see figure 6 below). These functions are chosen by MATLAB as standard when choosing fitting tool.
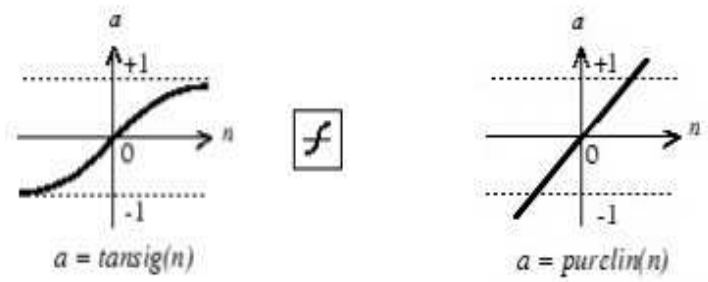

Fig..6. Transfer function Tan Sigmoid (left) and Linear (right).

C. Wind velocity

The wind velocity is needed to calculate the output energy of the wind turbine. The estimation is obtained by a network trained with the data from the year 2010 and the functions mentioned above. For ease of presentation purposes, oone day instead of one week of estimation is made. Estimation together with actual data of one day during the year 2011 is illustrated in Fig. 7 for the wind velocity in $\mathrm{km} / \mathrm{h}$. The wind speed is very variable and the estimation errors are expected to be higher compared to the previous cases. 


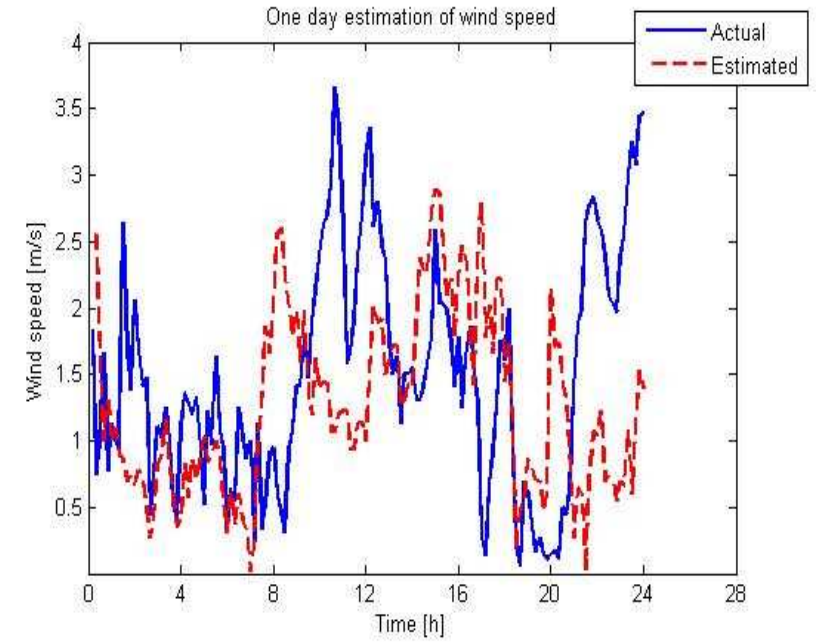

Fig. 7. One day of wind speed, estimated and actual data.

As mentioned before, it is important for the system to know how much electricity the house consumes. The estimation is done by a network trained with the data from the year 2010 and the functions mentioned above. Estimation together with actual data of one week during the year 2011 is illustrated in Fig. 8 for the electricity consumption (in W1Omin). The electricity consumption is probably the smallest error, since the consumptions do not change so much from day to day.

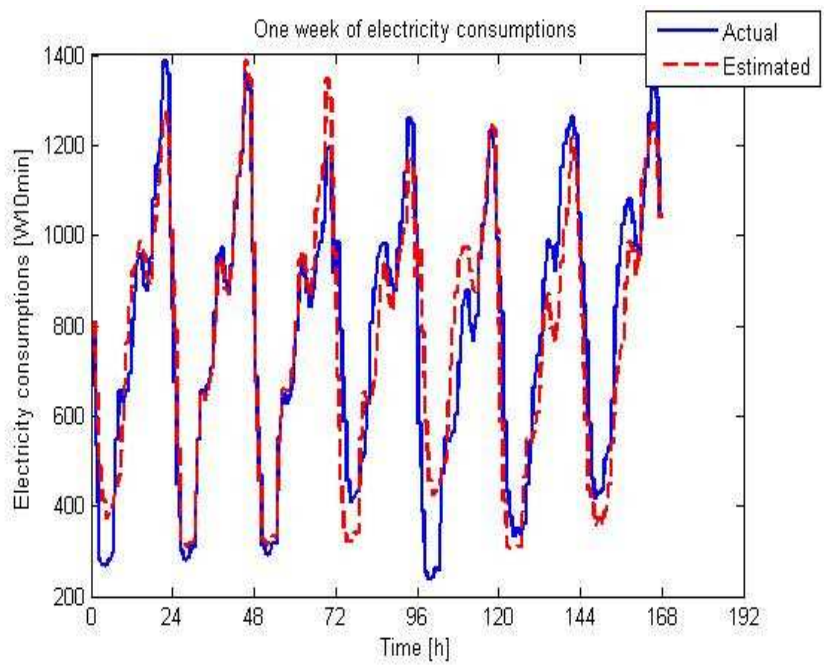

Fig. 8. One week of electricity consumption, estimated and actual data.

\section{Conclusions}

As it was shown the estimation quality varies from day to day, while slower weather changes gives a good estimation and rapid weather changes gives less good estimation as is normally expected. A better estimation results in a better performance, meaning that if more variables like clouds movement, wind velocity, air pressure, temperature, precipitation etc. are included then the more complex the model that needs more computation power.

The results of the neural network demonstrate the feasibility of the proposed approach, using an ANN to identify patterns in the wind speed as a microrenewable source and in the electricity consumption in residential areas. Additionally, we successfully identified patterns and showed that forecast of wind speed and electricity consumptions conditions can be done as part of the Energy Box concept by using a neural network.

Considering that the presented work is part of a larger and more complicated algorithm that the aggregator (i.e., energy box) contains, a neural network can be used to obtain a similar probability density function for forecasting of other parameters (i.e., solar). For solar electricity production forecasting, the neural network use can be very similar to the one used for wind production. Because the wind conditions are much more stochastic than the solar, it would be possible to obtain a neural network with a better performance and the final probability density function would have a lower variance. Other parameters, that serve as an input to the energy box such as electricity prices, and occupancy patterns and are part of future work can be also predicted with simpler neural networks, or any other classification, like support vector machines. In the context of an Energy box integrated in a microgrid, the possibility of identifying patterns in the solar behaviour for residential solar panels is very important, because it allows the energy box to enhance the decision making process, by increasing the quality of the inputs.

\section{References}

[1] M. A. Pedrasa and T. Spooner, "A survey of techniques used to control microgrid generation and storage during island operation", presented at the Australian Universities Power Engineering Conf., Dec. 10-13, 2006.

[2] A. Niniisto, Simulation of the Management of a Microgrid with Wind, Solar and Gas Generators, Aalto University School of Science and Technology, October 2009.

[3] S. Rahman, "An introduction to microgrid for integrated distributed generation and energy efficiency applications", Virginia Tech, November 2009.

[4] M. Stiebler, Wind Energy Systems for Electric Power Generation, Berlin, 2008.

[5] "Anern 1000L Wind Turbine", 2012-12-20, available: http://www.alibaba.com/product-gs/529131207/1KW _Residential_Wind_Turbines_for_the.html

[6] Available at http://www.energy.eu/

[7] "Iberdrola electric utility", 2011-11-10, Available at https://www.iberdrola.es/webibd/corporativa/iberdrola?IDP AG=ESWEBCLIEMPASEINFSUMTAR

[8] Evolución de las tarifas eléctricas", 2011-11-22, Available at http://javiersevillano.es/TarifasElectricasEvolucion.htm

[9] Mark Hudson Beale, Martin T. Hagan, Howard B. Demuth, "Neural Network Toolbox ${ }^{\mathrm{TM}}$ User's Guide", 2011, available at:

http://www.mathworks.com/help/pdf_doc/nnet/nnet_ug.pdf

[10] Available at http://www.euskalmet.euskadi.net 